\title{
DIFFERENTIAL OPERATOR STURM-LIOUVILLE TYPE ON THE SEGMENT $[0, \pi]$ WITH VARIABLE DELAY
}

\section{FATIH DESTOVIĆ ${ }^{1}$, ISMET KALČO ${ }^{2}$ and FIKRET ČUNJALO ${ }^{1}$}

${ }^{1}$ Faculty of Natural Sciences

University of Sarajevo

Sarajevo

Bosnia and Herzegovina

${ }^{2}$ Faculty of Polytechnic

University of Zenica

Zenica

Bosnia and Herzegovina

e-mail: hanasim@windowslive.com

\begin{abstract}
This paper studies the differential equation of the second order in the segment $[0, \pi]$ with varying delays. Construct a characteristic function operator a generated this equation and the boundary conditions at the ends of space, studying certain generalization of these operators, which naturally imposed.
\end{abstract}

2010 Mathematics Subject Classification: 35Qxx.

Keywords and phrases: characteristic functions, spectrum analysis, asymptotic functions, eigenvalues.

Received January 25, 2018; Revised March 27, 2018

(C) 2018 Scientific Advances Publishers 


\section{Introduction}

Spectral analysis is a modern mathematical theory that has proved to be extremely effective in addressing a much broader class of problems from different disciplines such as mathematics, mechanics, physics, electronics, geophysics, meteorology, and other natural and technical sciences. Reverse spectral problems today represent one of the most popular parts of the spectral analysis which is supported by a large number of papers devoted to just this issue [8] and [9]. The greatest results in the spectral theory in general and specifically to inverse spectral problems has been made from integral Sturm-Liouville operator

$$
L[y]=-y^{\prime \prime}(x)+q(x) y,
$$

which is also called one-dimensional Schrödinger operator.

This mathematical model is found in the application of mathematics, mechanics, electronics, and other sciences [1] and [4]. This class of differential operators describing the physical phenomena that do not depend only from the moment they are happening already on them is significantly affected by what happened before that moment. One such problem is the subject of this paper.

The spectral characteristics of the operator $L[y]$ implise asymptotic study of its intrinsic value, its asymptotic eigenfunctions, decomposition in order eigenfunctions, solving tasks and reverse the determination regular traces. A special class of inverse problems are problems for operators of Sturm-Liouville type where the potential $q(x)$ and the argument of the function $y$ does not occur with the same argument. Differential equations that describe these operators are called differential equations with shifted argument.

Definition 1. Differential equations whit shifted argument it is a differential equation in which the unknown function and its running appear for different values of the argument. 
Besides a number of boundary value problems which are studied and published the results of research, studied the following boundary problem:

$$
\begin{gathered}
-y^{\prime \prime}(x)+q(x) y(x-\tau)+\sum_{i=1}^{k} p_{i}(x) y\left(\beta_{i}\right)=\lambda y(x), \\
y(0)=y(\pi)=0, \\
y(x-\tau)=0 ; x \leq \tau .
\end{gathered}
$$

So, the initial set of the segment $[-\tau, 0]$, that for $\tau>0$ can not be reduced to the point. This condition describes the phenomena that occur in missile technology and ballistics, just before firing the missiles, and the factors that affect the speed and trajectory as well as the phenomena in geophysics at the stress and displacement of tectonic plates.

\section{Generalization Operators}

In this paper, we consider the following equations:

$$
\begin{aligned}
-y^{\prime \prime}(x)+q(x) y(\alpha(x))+ & \sum_{i=1}^{k} p_{i}(x) y\left(\beta_{i}\right)=\lambda y(x)=z^{2} y(x), \\
y(0) & =0 \\
y(\pi) & =0
\end{aligned}
$$

whereby the operator of $p_{i}(x) \equiv 0, x \in[0, \pi]$ discussed in [7] and is constructed its characteristic function. The initial set is reduced to the point $x=0$. The problem boils down to solving the following equation, the resolution of which are presented in the form of the following theorem.

Theorem. If the function $q(x), p_{i}(x),(i=1,2, \ldots, n) \alpha(x)>0$, $0<\alpha^{\prime}(x)<x, 0<\alpha(x)<x$. For each $x \in(0, \pi], \alpha(0)=0, x \in[0, \pi]$ infinitely differentiable functions and some are $\beta_{i}(x)(0, \pi), i=1,2, \ldots, k$ 
set but arbitrary numbers. Then the characteristic function $F_{\beta_{1}}(z)$ the operator of the present (1)-(3) meromorphic function whose poles zero function.

Proof. Let

$$
y(x, z)=2 i C \sin z x+\frac{1}{z} \int_{0}^{x} q(t) \sin z(x-t) y(\alpha(t)) d t+\sum_{i=1}^{k} y \beta_{i} \int_{0}^{x} p_{i}(t) \sin z(x-t) d t,
$$

where $C$ is a constant, $i=\sqrt{-1}$ and let

$$
h_{i}(x, z)=\sin z x+\int_{0}^{x} p_{i}(t) \sin z(x-t) d t ; \quad i=1,2, \ldots, k,
$$

without limiting the generality we can put $2 i C=1$.

So we come to the integral equation.

$$
y(x, z)=\sin z x+\sum_{i=1}^{k} \frac{1}{z} h_{i}(x, z) y\left(\beta_{i}, z\right)+\frac{1}{z} \int_{0}^{x} q(t) \sin z(x-t) y(\alpha(t)) d t .
$$

We'll put

$$
y_{0}(x, z)=\sin z x+\sum_{i=1}^{k} \frac{h_{i}(x, z)}{z} y\left(\beta_{i}, z\right) .
$$

We will be considered as the size of $y\left(\beta_{i}, z\right), i=1, \ldots, k$ known sizes and Equation (4) can be solved by successive approximations. For $k=1$ follows:

$$
\begin{aligned}
y_{1}(x, z)= & {\left[\sin z x+\frac{1}{z} \int_{0}^{x} q(t) \sin z(x-t) y(\alpha(t)) d t\right] } \\
& +y\left(\beta_{1}, z\right)\left[\frac{h_{1}(x, z)}{z}+\frac{1}{z} \int_{0}^{x} q(t) \sin z(x-t) h_{1}(\alpha(t), z) d t\right]
\end{aligned}
$$




$$
\begin{aligned}
y_{2}(x, z)= & {\left[\sin z x+\frac{1}{z} \int_{0}^{x} q(t) \sin z(x-t) \sin z(\alpha(t)) d t\right.} \\
& \left.+\frac{1}{z} \int_{0}^{x} q(t) \sin z(x-t) \int_{0}^{\alpha(t)} q\left(t_{1}\right) \sin z\left(\alpha(t)-t_{1}\right) \sin z \alpha\left(t_{1}\right) d t_{1} d t\right] \\
& +y\left(\beta_{1}, z\right)\left[\frac{h_{1}(x, z)}{z}+\frac{1}{z^{2}} \int_{0}^{x} q(t) \sin z(x-t) h_{1}(\alpha(t), z) d t\right. \\
& \left.+\frac{1}{z^{3}} \int_{0}^{x} q(t) \sin z(x-t) \int_{0}^{\alpha(t)} q\left(t_{1}\right) \sin z\left(\alpha(t)-t_{1}\right) h_{1}\left(\alpha\left(t_{1}\right), z\right) d t_{1} d t\right] .
\end{aligned}
$$

Introduce the following tags:

$$
\begin{aligned}
\varphi(x, z)= & \sin z x+\sum_{i=1}^{\infty} \frac{1}{z^{i}}\left\{\int _ { 0 } ^ { x } q ( t ) \operatorname { s i n } z ( x - t ) \int _ { 0 } ^ { \alpha ( t ) } q ( t _ { 1 } ) \operatorname { s i n } z \left(\alpha(t)-t_{1} \int_{0}^{\alpha\left(t_{1}\right)} \ldots\right.\right. \\
& \left.\ldots \int_{0}^{\alpha\left(t_{i}\right)} q\left(t_{i+1}\right) \sin z\left(\alpha\left(t_{i}\right)-t_{i+1}\right) d t_{i+1} \ldots d t_{1} d t\right\}, \\
\psi(x, z)= & h_{1}(x, z)+\sum_{i=1}^{\infty} \frac{1}{z^{i}}\left\{\int _ { 0 } ^ { x } q ( t ) \operatorname { s i n } z ( x - t ) \int _ { 0 } ^ { \alpha ( t ) } q ( t _ { 1 } ) \operatorname { s i n } z \left(\alpha(t)-t_{1} \int_{0}^{\alpha\left(t_{1}\right)} \ldots\right.\right. \\
& \ldots \int_{0}^{\alpha\left(t_{i}\right)} q\left(t_{i+1}\right) \sin z\left(\alpha\left(t_{i}\right)-t_{i+1}\right) h_{1}\left(\alpha\left(t_{i+1}, z\right) d t_{i+1} \ldots d t_{1} d t\right\} .
\end{aligned}
$$

Then we have

$$
y(x, z)=\varphi(x, z)+\frac{1}{z} y\left(\beta_{1}, z\right) \psi(x, z),
$$


where for $x=\beta_{1}$, we have

$$
y\left(\beta_{1}, z\right)=\frac{\varphi\left(\beta_{1}, z\right)}{1-\frac{1}{z} \psi\left(\beta_{1}, z\right)} .
$$

Therefore,

$$
y(x, z)=\varphi(x, z)+\frac{\varphi\left(\beta_{1}, z\right)}{z-\xi\left(\beta_{1}, z\right)} \xi(x, z)
$$

Since $\varphi(0, z)=0$ and $\xi(0, z)=0$, it is a condition of $y(0)=0$ in function (5) already installed. So the characteristic equation of the boundary problem looks like

$$
\varphi(\pi, z)+\frac{\varphi\left(\beta_{1}, z\right)}{z-\xi\left(\beta_{1}, z\right)} \xi(\pi, z)=0
$$

Thoroughly investigate the asymptotic left Equation (6). Asymptotic function $\varphi(x, z)$ is given in, and the asymptotic function $\xi(x, z)$ in the case of constant delay. As in the case of our equation acting analogously we get:

$$
\xi(x, z)=\sum_{i=0}^{\infty} \frac{\psi_{2 i+1}(x)}{z^{2 i+1}}+\sin z x \sum_{\nu=1}^{\infty}\left[\frac{\psi_{2 \nu}}{Z^{2 \nu}}\right]+\sum_{j=0}^{\infty} \cos z \alpha_{j}(x) \sum_{\nu=1}^{\infty}\left[\frac{\psi_{j, \nu}^{(c)}(x)}{z^{\nu}}\right] .
$$

where $\alpha_{j}(x)=\alpha(\alpha \ldots \alpha(x) \ldots), \quad j=0,1, \ldots, \alpha_{0}(x)=x$.

For the sake illustration, said the first few coefficients

$$
\begin{gathered}
\Psi_{1}(x)=p_{1}(x) \Psi_{0,1}^{(c)}=-p_{1}(0), \\
\Psi_{3}(x)=-p_{1}^{\prime \prime}(x)+q(x) p(\alpha(x)) \Psi_{0,2}^{(c)}=0 \\
\Psi_{0,3}^{(c)}=-p_{1}(0) q(0)\left[1+\frac{\alpha^{\prime}(0)}{\alpha^{\prime 2}(0)-1}\right], \quad \Psi_{1,3}^{(c)}=\frac{p_{1}(0) q(0)}{\alpha^{\prime 2}(x)-1}, \quad \Psi_{2}^{(3)}=-p_{1}(0) .
\end{gathered}
$$


We'll take the equity out of (4) in the form of something exchanged between a

$$
\begin{array}{cc}
\xi(x, z)=\sum_{j=0}^{\infty} \sin \alpha_{j}(x) z\left[\frac{1}{z^{j}} \sum_{\nu=0}^{\infty} \frac{A_{j \nu}(x)}{z^{\nu}}\right], \\
A_{0,0}(x)=1, & A_{1,0}(x)=0, \\
A_{0,1}(x)=0, & A_{1,1}(x)=0, \\
A_{0,2}(x)=-\frac{q(0)}{1-\alpha^{\prime 2}(0)}, & A_{0,2}(x)=\frac{q(x)}{1-\alpha^{\prime 2}(x)} .
\end{array}
$$

Putting $x=\pi$ and $x=\beta_{1}$ in (7) and (8) and then by integrating the (6) we obtain the asymptotic characteristic function. Characteristic function is meromorphic function variable $z$, where $z$ zero function $z-\zeta\left(\beta_{1}, z\right)$ poles characteristic function. Following the reasoning of [7], the characteristic function $F_{\beta_{1}}(z)$ can be considered as the entire function. Also, the reasoning of [7] for the case $k>1$ is here entirely feasible and conclusions are analogue.

\section{Conclusion}

In this work, I watched the inverse problem for the Sturm-Liouville's operator with outspread argument, I've built the characteristic function for the differential operator tenders, a completely new equation. Results previously proven theorems apply to the operator given by (9)

$$
\begin{gathered}
-y^{\prime \prime}(x)+\sum_{i=1}^{k} q_{i}(x) y\left(\alpha_{i}(x)\right)-\sum_{j=1}^{k} p_{j}(x) y\left(\beta_{j}\right)=\lambda y(x), \\
y(0)=y(x)=0 .
\end{gathered}
$$

This item analysis and an improvement in comparison with the concept of "ideal" process which is obtained under the assumption that after the 
fact does not. This assumption generally well reflects the real world phenomena as in cases where the delay is related to the submission of a sound signal, with hydraulic shock wave or other processes. This mathematical modelling of differential operators with constant, homogeneous delay as and in this paper.

\section{References}

[1] G. Freiling and V. Yurko, Inverse Sturm-Liouville Problems and their Applications, Nova Science Publishers, Inc., Huntington, New York, 2008.

[2] V. Ambarzumjan, Uber eine frage der eigenwerttheorie, Zeitschrift für Physik 53(9-10) (1929), 690-695.

$$
\text { DOI: https://doi.org/10.1007/BF01330827 }
$$

[3] С. Б. Норкин, Дифференциальнюе уравнения второго лорядка с запаздывающим аргументом, Наука, Москва, 1965.

[4] N. Levinson, The inverse Sturm-Liouville problem, Matematisk Tidsskrift. B (1949), 25-30.

[5] O. H. Hald, Discontinuous inverse eigenvalue problems, Comm. Pure Appl. Math. 37(5) (1984), 539-577.

DOI: https://doi.org/10.1002/cpa.3160370502

[6] R. Carlson, Inverse spectral theory for some singular Sturm-Liouville problems, J. Differential Equations 106(1) (1993), 121-140.

DOI: https://doi.org/10.1006/jdeq.1993.1102

[7] M. Pikula, Asymptotic Eigenvalues and Regularized Traces of Linear Differential Operators Doctoral Dissertation, Sarajevo, 1983.

[8] Б. М. Левитан and И.С. Саргсян, Операторы Штурма-Лиувилля и Дирака, Москва, Наука, 1988.

[9] A. Bayramov, S. Ozturk Uslu and S. Kizilbudak Caliskan, Computation of eigenvalues and eigenfunctions of a discontinuous boundary value problem with retarded argument, Appl. Math. Comput. 191(2) (2007), 592-600.

DOI: https://doi.org/10.1016/j.amc.2007.02.118

[10] E. Bas and R. Ozarslan, Sturm-Liouville problem via coulomb type in difference equations, Filomat 31(4) (2017), 989-998.

DOI: https://doi.org/10.2298/FIL1704989B 